\title{
Innovative methods for forming concepts of object size and measurement of sizes in preschool children \\ ${ }^{1}$ Ostanov Kurbon
}

Candidate of pedagogical sciences, associate professor, Department of Probability Theory and Mathematical Statistics,

${ }^{2}$ Mardanov Eshim Muratovich

Candidate of Pedagogical Sciences, Associate Professor,

Department of Theory and Methodology of Preschool Education

${ }^{3}$ Kadirov Jasur

Assistant of the Department of Theory and Methods of Preschool Education

${ }^{1,2,3}$ Samarkand State University, Samarkand

\begin{abstract}
The article analyzes various approaches to the content and methods of forming ideas about the size of an object and measuring values in preschool children, as well as technologies for forming the ability to use the correct names of specific lengths and correctly show them, when comparing 2 objects by weight (3 - 5 years) first (junior age) in situations of everyday life, in the process of communication and games, the words "heavy, light", "heavier, lighter" are introduced into the active vocabulary of children, methodical work on the formation of skills to arrange more than 2 objects according to size and weight (2 - 6 years) and provides recommendations for their implementation in practice.

Key words: technology, ideas, object size, measurement of quantities, objects, masses, thickness, width, length, size, comparison.
\end{abstract}

\section{Introduction.}

When forming ideas about the size of an object and measuring values in preschool children, an important role is played by the formation of their ability to use the correct names of specific lengths and correctly show them (up to 4 years). In this case, the Educator himself must always use the name of specific dimensions to indicate the size of the object. For children of primary preschool age, visual material on the basis of comparison should be very contrasting. Display techniques include: the length is usually shown from left to right horizontally (or by meaning). The width should be shown from bottom to top along the sagital (transverse axis). Height - from bottom to top along the vertical. Depth - from top to bottom vertically. Thickness - along the circumference of the section.

A note about the attribute "thickness". Proceeding from the fact that thickness is associated with diameter, and rounded objects have diameter, then in order to form ideas about thickness it is necessary to take objects of a rounded shape, preferably cylindrical. Objects of the shape of a rectangular parallelepiped are said to have length, width, height, i.e. do not have a thickness. If objects have 2 extensions: length (or height) and thickness, then they are suitable for forming ideas about thickness. Flat geometric shapes have no thickness. If we assume that we have "thickened" the circle, then it will be a cylinder with a certain height.

\section{Research results.}

Flat objects have only length and width, but no height, but a planar image of volumetric objects can have a height. At the next stage, work is underway to develop the ability to compare 2 objects in length, width, height, thickness using application and overlay techniques (3 - 4 years). This is where the app trick is used first. Algorithm for comparing objects by size (for example, by length): children are offered 2 identical objects for all characteristics, except for the one being compared (except for length); it becomes clear how objects differ; it is proposed to check which item, for example, is longer; for this, objects must be positioned so that they touch according to a comparable feature: lengthwise widthwise heightwise thickness; items are trimmed on one side; reasoning about the presence or absence of an extra piece is conducted, the conclusion is drawn: the strip that has an extra piece is longer, if the piece is not enough, it is shorter; the length is shown with the arms outstretched (using the motor analyzer); finally, children are offered situations and exercises in which it is necessary to compare objects according to one of the signs.

The overlay technique is usually used to compare flat objects in length or width, or to compare flat images of threedimensional objects. The comparison methodology is the same as for receiving an application with the difference that: the objects must necessarily differ in color; objects overlap each other: in width in length. All other points are the same as in the application.

In middle age, children are taught to compare objects at once according to 2 signs (for example, length and width), but first one of the signs must be the same for two objects, for example: find a ribbon of the same length, but wider. Then we propose to compare different objects in two different ways. At an older age, children are taught to compare objects along 
three dimensions at once.

When comparing 2 objects by weight ( 3 - 5 years), first (younger) in situations of everyday life, in the process of communication and games, the words "heavy, light", "heavier, lighter" are entered into the active vocabulary of children. The meaning of these words is explained. Situations such as moving furniture, swinging, playing boats are used (boats are bars of different weights). For children of the older group, teach them how to compare 2 objects by weight, using the method of imitating the movement of the beam balance by hands.

Weighing rules: we take objects in our hands, palms up, straighten our palms, perform the movements of the lever scales with our arms slightly outstretched forward. Determine in which hand the object is heavier; swap items and repeat weighing. We conclude: "the red cube was heavier in both the right and left hand, so it is heavier than the blue one".

Visual material should be excellent only in comparison with the characteristic and color. Visual material options: objects made of material of different densities; - hollow objects into which something is poured or a weight is placed. The problem is solved on an individual basis, because the mass is invisible.

Methodical work on the formation of skills to organize more than 2 items by size and weight (2-6 years) at the initial stage (2-3 years.). Children play with nesting dolls and other in-place toys by trial and error. With children aged (4-5 years old). A special ordering method is shown. First, exercises are given to find the place of an object among others in a readymade ordered row. Then it is proposed to arrange objects according to a given criterion: first, according to a model, then according to verbal instructions (from the shortest to the longest). First, exercises are given to build increasing in size, and then decreasing series. In the wording of the tasks, not only the order of ordering, but also the direction of laying out the objects (from bottom to top, from left to right) must be indicated.

With children from the age of 5 , they are taught to arrange more than 5 objects by length, width, height, thickness and compare 3 objects by mass. The contrast in size decreases. Learn to order using the pairwise comparison method (application or weighing). For example, you need to arrange 3 balls by mass: red, yellow, green. Suppose we need to arrange the balls from the lightest to the heaviest from left to right. We set the task: choose the lightest of all balls. To do this, we take any 2 balls and compare them by mass; from them we choose the one that is easier; we leave it in our hands, and put the second aside, take the 3rd ball and compare with the one in the hand. Again, from 2 balls, choose the one that is lighter and put it first on the left - "the lightest ball"; then again choose the lightest of the remaining balls. Which is lighter, we put the second, the rest we put the last, it is "the heaviest".

\section{Conclusions.}

Formation of the ability to compare objects in three dimensions ( 5 - 6 years). ... Teach children to show all 3 dimensions (height, length, width) on one object. Initially, objects are used that occupy a constant position in space (furniture). Then we use objects that change their position in space (building material, boxes, crafts). Then children are taught to compare objects in terms of volume as a whole (in 3 dimensions). Objects must be such that all measurements of one of them are greater than the corresponding parameters of the other. For example: a wardrobe in a dressing room for children and adults. We measure length, width, height with a conventional measure. If the length of the adult wardrobe is greater than the length of the children's wardrobe and the width of the adult wardrobe is greater than the width of the children's wardrobe, and the height is the same, then the adult wardrobe is larger than the children's wardrobe. Exercises are offered to arrange the same objects, first one by one, then by another criterion, and then by volume as a whole.

Formation of an understanding of the immutability (preservation) of the size of an object (mass, length, area, volume) when changing its shape (5 - 6 years). This problem is solved in the classroom for visual activities. Children are offered 2 objects of the same size and shape. Then, before the eyes of the children, the shape of one object changes. The teacher emphasizes that nothing was added or subtracted to the object. It turns out what has changed and what has remained the same. It is concluded that the size of the object has not changed. The children are shown the proof of this in one of the ways: we give both objects the same (original) shape. we measure objects.

Consider how to show children the independence of the mass of an object from changing its shape. We offer 2 objects of the same weight and shape (two balls of plasticine). Then, in front of the eyes of the children, the shape of one object changes (a cake is made). The teacher emphasizes that they did not add or subtract plasticine. Questions are asked: "What has changed, and what has remained the same?", "Has the mass (the amount of plasticine) changed?" The proof is shown that the mass has not changed: both objects are given the same (original) shape. You can weigh objects on a beam balance (if available in kindergarten).

\section{References}

1. Fiedler, Maria. Mathematics already in kindergarten: A manual for a kindergarten teacher / Maria Fidler; translated from Polish by O. A. Pavlovich. - Moscow: Education, 1981 .-- 159 p. :

2. Montessori M. Maria Montessori. Children are different. A unique technique for early development. - M .: AST, 2015

3. Pavlova L.I. Theory and methodology for the development of mathematical representations in preschoolers / L.I. Pavlova - M.: Moscow State Pedagogical University, 2017 .-- 108 p. 
4. Beloshistaya AV Formation and development of mathematical abilities of preschool children: Questions of theory and practice: A course of lectures for students. doshk. faculties of higher. study. institutions. - M .: Humanit. ed. center VLADOS, 2003 - 400 e .:

5. Mikhailova A., Nosova E. D., Stolyar A. A., Polyakova M. N., Verbenets A. M. Theory and technology of mathematical development of preschool children. Publishing house "Childhood-press". St. Petersburg, 2008.

6. Glenn Doman, Janet Doman How to increase your child's intelligence. 2015 Publisher: MEDIAKIT Series: Soft Revolution

7. Ostanov, K., Inatov, A., Himmatov, I., \& Rakhimov, B. (2018). ON SOME WAYS OF DEVELOPING THE CREATIVE ACTIVITY OF STUDENTS WHEN SOLVING EQUATIONS. BBK 72 S108.

8. Ostanov, K., Inatov, A., \& Himmatov, I. (2018). ROLE OF THE SYSTEM OF KEY TASKS IN THE PROCESS OF TEACHING MATH. In EUROPEAN RESEARCH: INNOVATION IN SCIENCE, EDUCATION AND TECHNOLOGY (pp. 77-79).

9. Ostanov, K., Inatov, A., Abdurakhmonova, M., \& Shamsieva, G.A. (2018). ON SOME WAYS OF DEVELOPING THE THINKING OF STUDENTS IN THE PROCESS OF SOLVING GEOMETRIC PROBLEMS. BBK 72 A105.

10. Ostanov K., Khayitmuradov Sh., Murtazaev M. ABOUT THE FORMATION OF RESEARCH SKILLS IN STUDENTS WHEN STUDYING THE LOCATION OF THE ROOTS OF THE SQUARE EQUATION // INNOVATIVE PROCESSES IN THE SCIENTIFIC ENVIRONMENT. - 2019 .-- S. 350-353. 